\title{
Characterisation of time-dependent, statistical failure of cellulose fibre networks
}

\author{
Amanda Mattsson (1D) Tetsu Uesaka
}

Received: 15 February 2018/ Accepted: 2 April 2018/Published online: 13 April 2018

(C) The Author(s) 2018

\begin{abstract}
Cellulosic materials have special advantages for transport packaging, because of their lightweight and recyclable natures and also relatively high specific strength. The strength of such materials is normally evaluated by applying monotonically increasing, quasi-static displacement (or load). However, in real circumstances, the material is subjected to far more complex loading histories, such as creep, fatigue, and random loading. Failures under such circumstances are, not only time-dependent, but also notoriously variable. For example, the coefficient of variation for creep lifetime reaches or even exceeds $100 \%$. The objective of this study is to develop a method to characterise both time-dependent and statistical natures of failures of cellulosic materials. We have used a general formulation of time-dependent, statistical failure, originally proposed by Coleman (J Appl Phys 29(6):968-983, 1958). We have
\end{abstract}

identified three material parameters: (1) characteristic strength, representing short term strength, (2) brittleness parameter (or durability), and (3) Weibull shape parameter related to long-term reliability. These parameters were determined by special protocols of creep and constant loading-rate (CLR) tests for a series of containerboards. Results have shown that these two test methods yield comparable values for the materials parameters. This implies the possibility of replacing extremely time-consuming creep tests with the more time-efficient CLR tests. Comparing the cellulose fibre networks with fibres and composites used for advanced structural applications, we have found that they are very competitive in both reliability and durability aspects with Kevlar and glass-fibre composites.

\footnotetext{
A. Mattsson $(\bowtie) \cdot T$. Uesaka

Department of Chemical Engineering and FSCN, Mid Sweden University, Holmgatan 10, 85170 Sundsvall, Sweden

e-mail: amanda.mattsson@miun.se
} 


\section{Graphical abstract}

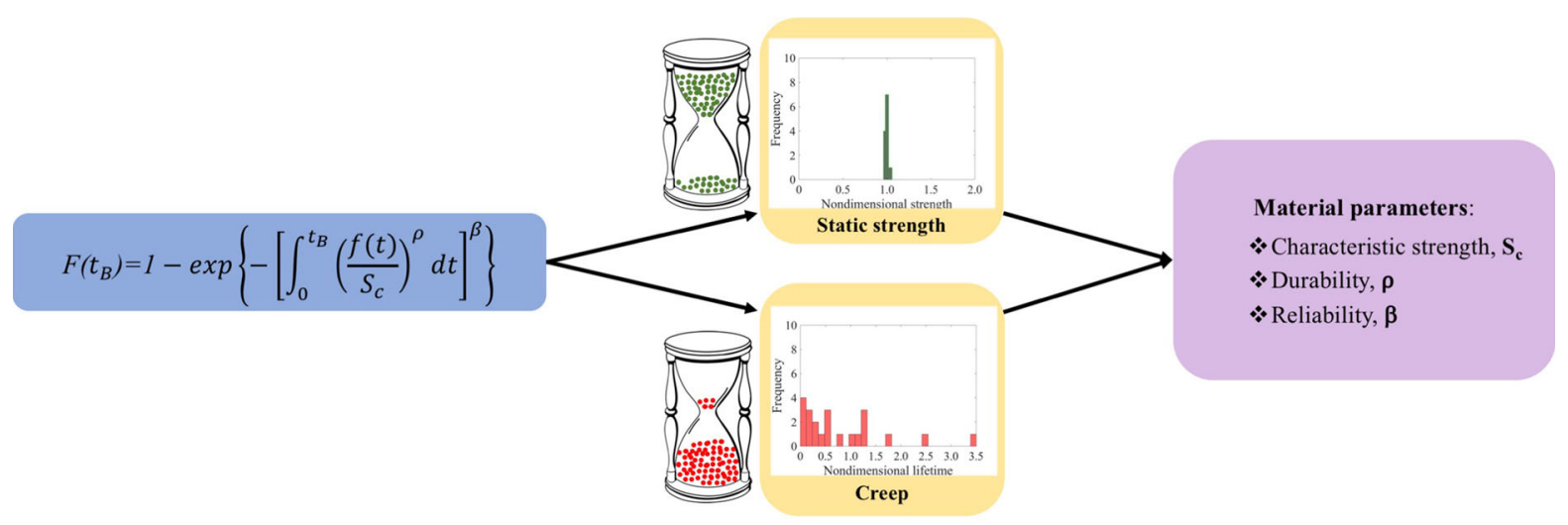

Keywords Time-dependent failure $\cdot$ Statistical failure $\cdot$ Fibre network $\cdot$ Strength $\cdot$ Creep · Characterisation · Durability $\cdot$ Reliability

\section{Introduction}

Material strength is traditionally evaluated by critical stress at which the material fails under monotonically increasing displacement (or load) conditions. However, it is known that materials often fail even at a much lower stress level, if it is subjected to stresses over a prolonged period, e.g., creep and fatigue conditions. Material strength is generally time-dependent or loading-history dependent. An interesting question may be "Does short-term strength predict long-term strength?".

Figure 1 shows an example of creep failure of corrugated boxes in compression, tested by Nyman (2004). (The work is based on his PhD work, and the authors received the data as his courtesy).

Prior to creep tests, ordinary compression tests (Box Compression Test, BCT) were performed for Box A and Box B, both of which are made of B-flute with the same size and configuration. Results showed that Box B was stronger $(2.92 \mathrm{kN})$ than Box A $(2.44$ $\mathrm{kN})$. However, creep strength, as measured by time-tocollapse (lifetime), showed the opposite: Box A had a longer lifetime than Box B (Fig. 1a). In other words, Box $\mathrm{A}$ is more durable than Box $\mathrm{B}$, even though the "ordinary" strength is lower. This poses a fundamental question of the relevance of typical static strength to longer-term strength properties. Another important aspect of long-term performance is variability. Each data point in Fig. 1a is, in fact, an average of 10 measurements of creep lifetime. Its scatter is plotted at each load level in Fig. 1b. The variations of lifetimes among the boxes were extremely large, and the coefficient of variation (COV) varied from 34 to $77 \%$ for the different load levels, although the measurements were done under a nominally constant environmental condition.

Similar large scatters of box creep lifetime were also reported in the literature from early days (e.g., Kellicutt and Landt 1951; Stott 2017; Moody and Skidmore 1966; Koning and Stern 1977; Popil and Hojjatie 2010). This large uncertainty of lifetime is a source of overdesign (i.e., taking higher safety factor) of boxes which are subjected to creep or long-term loading. Therefore, an important question is how to evaluate this multi-faceted nature of long-term strength, particularly the durability aspect, and also the uncertainty (or conversely, certainty or reliability) aspect, in a general framework.

In the literature, time-dependent failure has been dealt with in the areas of fatigue strength and creep strength (e.g., Murakami and Endo 1994; Wilshire 2002) for many years. Because of its complexity of underlying mechanisms, the approaches are largely empirical and phenomenological, but a few important empirical laws has been found (e.g., Miner 1945; Hashin and Rotem 1978; Monkman and Grant 1956), which are widely used for organising fatigue and creep strength data and also provided insights for the later development of damage evolution models [e.g., Curtin and Scher 1997]. The basic limitation of these approaches is, however, that they are mainly 
(a)

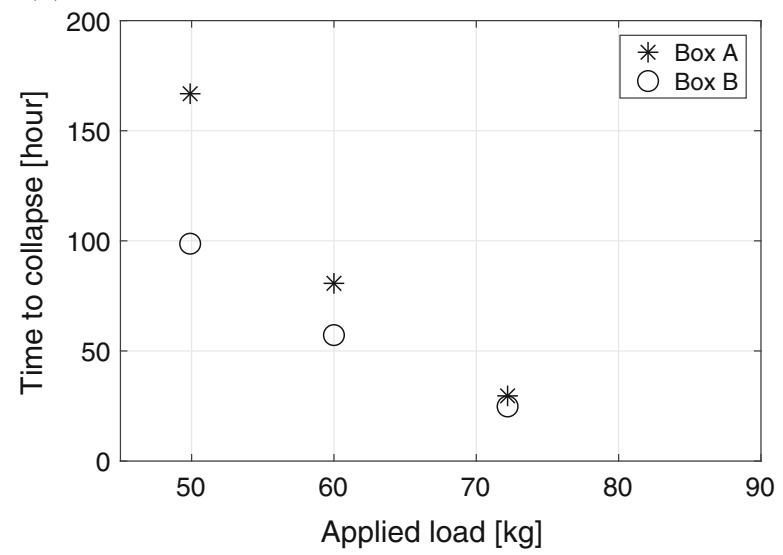

Fig. 1 a Time-to-collapse versus applied load for two different corrugated boxes. Each value of time-to-collapse is an average over 10 samples. Box compression test (BCT) result for box A is

deterministic, and thus there is no systematic treatment of the huge scatter inherent to fatigue and creep strength. In the area of cellulosic materials, the subject has been actively investigated as creep failure of container box under constant or cyclic humidity conditions (Kellicutt and Landt 1951; Stott 2017; Moody and Skidmore 1966; Koning and Stern 1977; Bronkhorst 1997; Kirkpatrick and Ganzenmuller 1997; Popil and Hojjatie 2010). Although enormous scatters of creep lifetime data were recognised in the early literature, systematic treatment of time-dependent, statistical failure has been still in infancy for cellulosic materials (e.g., for review, Coffin 2011).

The first rigorous treatment of time-dependent, stochastic failure is, probably, Coleman's model (Coleman 1958). He considered a fibre subjected to a general loading history (not only creep and fatigue), and obtained an expression for lifetime distribution based on three postulates: (1) weakest-link scaling, (2) damage evolution laws (exponential or power laws), and (3) an algebraic form of probabilistic failure condition. In the cases of creep-type loading history and constant loading rate (CLR) history, the model predicts Weibull distributions for lifetime and strength distributions, respectively. Although Coleman's model is for "fibre" (a chain of breakable elements), Phoenix and coworkers extended Coleman's approach to a system of fibre bundles (a parallel arrangement of breakable elements) as a model for uniaxially-reinforced fibre-polymer composites (Phoenix 1978; Phoenix and Tierney 1983; Tierney 1982; Newman (b)

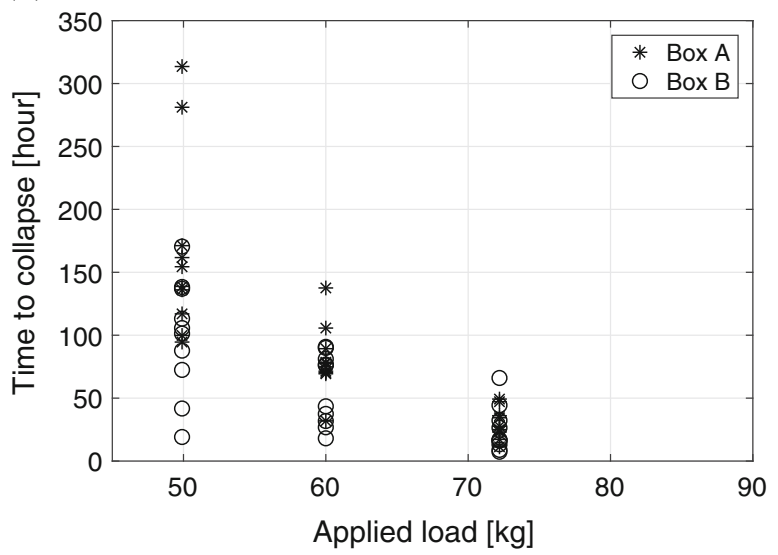

$2.44 \mathrm{kN}$, and for box B $2.92 \mathrm{kN}$. b Individual data points from the average time-to-failure in (a) against applied load

and Phoenix 2001; Mahesh and Phoenix 2004). The lifetime distributions of this system depend on the assumed load-sharing rules. In the case of the localload sharing rule (a more brittle system), it shows a non-Weibull distribution. The weakest-link-scaling behaviour appears only after the system size grows sufficiently large, unlike the fibre case. Christensen and Miyano $(2006,2007)$ took a different approach by considering the time-dependent growth of a single crack and treating critical stress as a random variable. Interestingly, the resulted expression for the relation between creep lifetime distribution and strength distribution was very similar to that obtained by Coleman.

In order to investigate how the time-dependent, statistical failure characteristics of fibre, as formulated by Coleman, is translated into the behavior of fibre network, the authors used a central-force, triangular lattice network and performed Monte-Carlo simulations of creep failure (Mattsson and Uesaka 2015, 2017). We found that the weakest-link scaling asymptotically appears with increasing the system size, as the fibre bundle model also showed (e.g., Newman and Phoenix 2001). Interestingly, the damage evolution law defined for fibre was preserved even in the fibre network level. The creep lifetime showed a distribution slightly deviated from Weibull distribution. We found that the resulted distribution is the same double-exponential form distribution, called DLB-type distribution, which was initially found for static strength of random fuse models (Duxbury et al. 1987) and later fibre bundle 
models (Wu and Leath 1999). However, it is important to note that, within a typical, experimentally-acceptable probability range (e.g., 0.01-0.99), the nonWeibull feature is subtle, thus very difficult to detect in a statistically significant manner. In other words, Weibull distribution approximately holds for lifetime distribution of fibre networks.

In conclusion, although the model proposed by Coleman for fibre is phenomenological, the basic relationship describing time-dependent, statistical failure is still preserved on the fibre network level. The only precaution is that the size dependency of the material parameters is different from that for typical Weibull distribution and the prediction of the lower tail of the distribution is always conservative because of the slight non-Weibull feature.

Therefore, in this study, we used Coleman's formulation to characterise different aspects of timedependent, statistical failure of cellulosic materials. As examples of cellulose fibre-based networks, we have tested commercial containerboards (liner and fluting used in for example corrugated boxes) to determine material parameters, and compared them with those from typical fibres and fibre-reinforced composites (which are normally used for more advanced applications).

\section{Theoretical background}

Coleman's general formula for the cumulative distribution function of lifetime, $F\left(t_{B}\right)$, is written as:

$F\left(t_{B}\right)=1-\exp \left\{-\left[\int_{0}^{t_{B}}\left(\frac{f(t)}{S_{c}}\right)^{\rho} d t\right]^{\beta}\right\}$

where $f(t)$ is load at time $t$, and $S_{c}, \rho$, and $\beta$ are material parameters, as we will describe shortly. This formula was originally derived by Coleman (1958), later generalised by Phoenix (1978), and also re-derived by Curtin and Scher (1997) based on a damageevolution model. The most important feature of this model is that one can take into account any loading history, such as creep, constant-rate loading, fatigue, random loading, etc. to determine lifetime distributions.

For example, for creep loading $f(t)=f_{0}=$ constant , the lifetime distribution is given by:
$F\left(t_{B}\right)=1-\exp \left\{-\left(\frac{f_{0}}{S_{c}}\right)^{\rho \beta} t_{B}^{\beta}\right\}$

From the above expression, one can derive various statistical quantities which are practically important. One of such parameters is median $\left(F\left(t_{B, \text { median }}\right)=1 / 2\right)$ :

$t_{B, \text { median }}=(\ln 2)^{1 / \beta}\left(\frac{f_{0}}{S_{c}}\right)^{-\rho}$

Although the mean of lifetime is often reported, it is difficult to accurately determine the mean in the case of lifetime, because of the extremely long tail of the lifetime distributions. Median is, on the other hand, an easy parameter to determine for a modestly large number of tests (e.g., more than 10). It can be easily shown from the above equation that taking the log of $t_{B, \text { median }}$ and plotting it against the log of applied load $\left(\log \left(f_{0}\right)\right)$ gives a linear relationship with its slope $-\rho$, as we will see later in Fig. 5b. This plot is used later to validate the applicability of Eq. 2 for describing the lifetime distribution.

For a constant loading rate (CLR) test, $f(t)=\alpha t$, where $\alpha$ is the loading rate, we can determine the distribution of strength $f_{B}\left(=\alpha t_{B}\right)$, by:

$G\left(f_{B}\right)=1-\exp \left\{-\left(\frac{1}{(\rho+1) S_{c}{ }^{\rho} \alpha}\right)^{\beta} f_{B}^{\beta(\rho+1)}\right\}$

In the same way, the mean of strength, $f_{B, \text { mean }}$, is given by:

$f_{B, \text { mean }}=\left\{(\rho+1) S_{c}^{\rho} \alpha\right\}^{\frac{1}{\rho+1}} \Gamma\left(1+\frac{1}{\beta(\rho+1)}\right)$

where $\Gamma$ is the gamma function. It can also be shown that taking the $\log$ of $f_{B, \text { mean }}$ gives a linear relationship with the $\log$ of loading rate $(\log (\alpha))$ with a slope of $1 /(\rho+1)$, which can be seen later in Fig. 7b. This relationship, again, can be used to examine whether the strength distribution follows Eq. 4.

As seen in these expressions (Eqs. 2, 4), the statistical failure responses in two different time scales (creep and static loading tests) are completely determined by the materials parameters, $S_{c}, \rho$, and $\beta$.

The parameter $S_{c}$, called characteristic strength, essentially represents short-term strength, i.e. it is the creep load at which approx. $63 \%$ of the samples fail 
within one unit time (second) in creep. (See Eq. 2. Note that $1-\exp (1)=0.63)$.

The parameter $\rho$ has a dual meaning: brittleness and durability. In Coleman's formulation (Coleman 1958), the parameter $\rho$ is defined in the damage evolution law:

$\frac{d \Omega}{d t} \propto c f(t)^{\rho}$

where $\Omega$ is a damage parameter. At a given force $f(t)$, the higher the $\rho$, the higher the rate of damage growth, i.e. the system becomes more brittle. Another meaning comes from its molecular interpretation given by Phoenix et al. (1988):

$\rho=\frac{U_{0}}{k T}$

where $U_{0}$ is a potential barrier for thermal fluctuations of atoms and $k T$ is its thermal energy with $k$, Boltzmann constant, and $T$, absolute temperature. As the potential barrier becomes comparatively higher than the thermal energy (the higher the $\rho$ ), less atoms go over the barrier and thus the system becomes more stable (more durable). Conversely, if temperature increases, then $\rho$ decreases and the material becomes more ductile (or less brittle).

The parameter $\beta$ is the Weibull exponent of lifetime distribution (Eq. 2): a higher value means less variation of lifetime, i.e., less uncertainty and thus more reliable. Therefore, from the material characterisation view point, it is the material property representing reliability of long-term strength (creep lifetime).

Among these parameters, characteristic strength, $S_{c}$, is the closest to a routinely measured property (static strength). Both durability, $\rho$, and reliability, $\beta$, aspects, are, however, largely overlooked in the characterisation of strength properties of cellulosic materials.

\section{Experimental characterisations}

Materials and methods

As typical examples of cellulose fibre networks, we have used commercial containerboard samples (liner and fluting used in for example corrugated boxes) of varying quality and basis weight. These samples were collected from board mills located in Sweden,
Germany, Austria, and Czech Republic. From these samples, test specimens were cut into the size of $105 \mathrm{~mm} \times 25 \mathrm{~mm}$ (the actual testing area between the clambs was $61 \mathrm{~mm} \times 25 \mathrm{~mm}$ ), both in the machine and the cross-machine directions, and conditioned at $23{ }^{\circ} \mathrm{C}$ and $50 \%$ relative humidity. Additionally, all samples were preconditioned at lower relative humidity in order to achieve the same equilibrium moisture content (TAPPI 2013). Prior to testing, samples were rigorously randomised by generating random numbers to obtain statistically homogeneous samples sets for the material parameter estimates. (See also the section "Characterisation of the material parameters, $S_{c}, \rho$, and $\beta$ "). These specimens were subjected to both compression creep tests and constant loading rate (CLR) compression tests, as described below.

Creep failure tests in compression

A series of compression creep tests have been performed under different applied loads. The time to failure are determined when the creep rate surpassed a certain threshold value. Typical creep failure curves are shown in Fig. 2a. We have used a long-span compression tester (LCT) with finger supports, which was built at Rise Bioeconomy (formerly Swedish Forest Products Research Laboratories, STFI). Testing procedures were detailed in Mattsson and Uesaka (2013). Applied loads have been varied on 3-4 levels, and at each load level 50-100 samples have been tested.

Constant loading rate (CLR) tests in compression

Although constant displacement rate (CDR) tests are more common in material testing, we have performed constant loading rate (CLR) tests in order to determine the characteristic parameters, as discussed earlier. The sample is compressed at a CLR until the failure occurs. The failure point was determined by setting a criterion of the relative decrease of the tangent modulus of the stress-strain curve. For this study, the ratio of the slope at failure to the initial slope was set to 0.05 . Some examples of the force-displacement curves are shown in Fig. 2b. The equipment used for compressing the containerboard specimens was originally designed at FPL (Forest Products Laboratory) in Madison, Wisconsin, USA (Fellers and Donner 2002), and we have rebuilt the equipment to fit to a MTS machine (see 
(a)

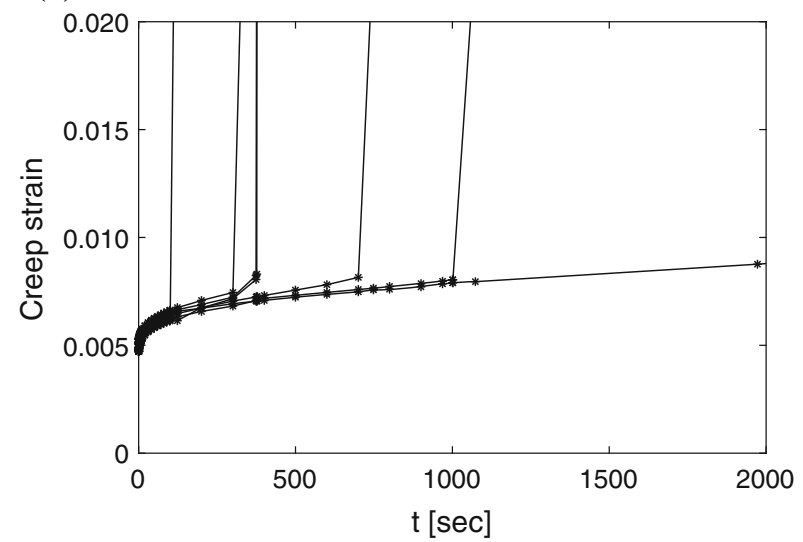

(b)

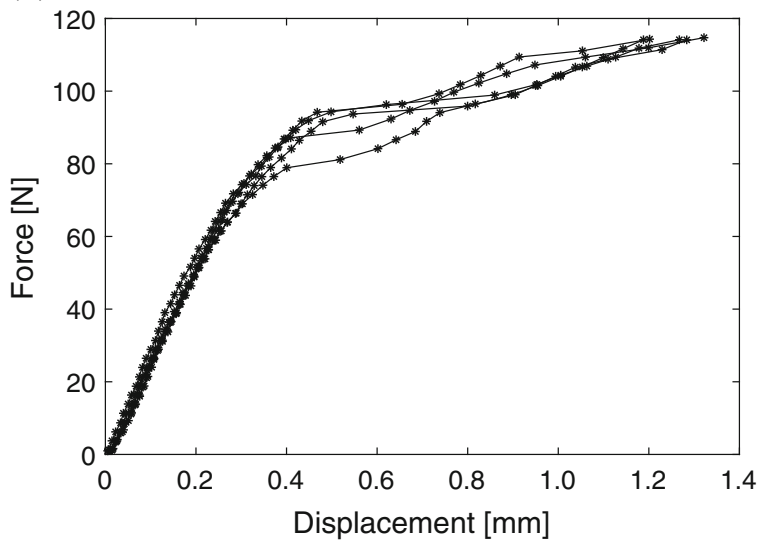

Fig. 2 a Creep strain versus time for five fluting samples of basis weight $140 \mathrm{~g} / \mathrm{m}^{2}$ (applied load: $56.0 \mathrm{~N}$ ). b Force-displacement curves for five samples of the same material as in (a) (loading rate: $1 \mathrm{~N} / \mathrm{s}$ )

Fig. 3). Loading rates have been varied on 4 levels, and at each loading rate, minimum 20 tests have been performed.

More detailed descriptions on the experimental procedures are available upon request.

Numerical determination of the parameters

In principle, the three material parameters, characteristic strength, Sc, brittleness parameter (or durability), $\rho$, and Weibull shape parameter, $\beta$, are determined by solving either Eq. 2 for creep tests or Eq. 4 for CLR tests. We have tried the maximum-likelyhood method,

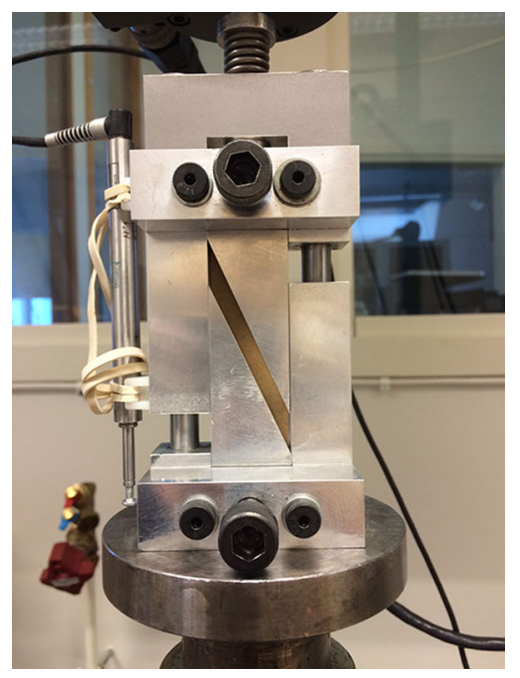

Fig. 3 Compression equipment used for performing the constant loading rate (CLR) tests within a MTS machine the direct nonlinear least-square solution of Eqs. 2 and 4 , and the nonlinear least-square solution of the same equations but in a Weibull format:

$$
\begin{aligned}
\log \left(-\log \left(1-F\left(t_{B}\right)\right)\right)= & \log \left(t_{B}\right) \beta+\log \left(f_{0}\right) \rho \beta \\
& -\log \left(S_{c}\right) \rho \beta \\
\log \left(-\log \left(1-G\left(f_{B}\right)\right)\right)= & \log \left(f_{B}\right) \beta(\rho+1) \\
& -\log \left(S_{c}\right) \beta \rho-\log (\rho+1) \beta \\
& -\log (\alpha) \beta
\end{aligned}
$$

where Eq. 8 represents creep, and Eq. 9 CLR tests, respectively. Because of the non-linear nature of the least-square equations, the first two approaches sometimes gave unstable solutions. Therefore, we have used the third approach to determine the material parameters. The numerical solver we have used is fitnlm (MATLAB 2017) in the MATLAB environment. The starting values (initial guess) for nonlinear fitting can be obtained by the Weibull plots based on Eqs. 8 and 9.

The estimation errors for these three parameters are also based on the above solution. Typical estimation errors, as expressed as relative standard errors, were very small, in the range of $0.03 \%$ for characteristic strength, and $2-6 \%$ for $\rho$ and $\beta$. However, during the course of this study, we have found large variations of these material parameters, from batch to batch and from position to position, for nominally the same sample (e.g., linerboard samples with the same basis weight, produced from the same mill, from the same 
board machine, and even at the same position across the machine). Accordingly, we have developed and perfected, progressively, a random sampling procedure over the same period. Unfortunately, this has caused some constraints to the sample-to-sample comparisons, particularly the comparison of results between creep tests and CLR tests, since the latter were conducted at a later phase of the entire project period of 5 years.

\section{Results and discussion}

Time dependent failure/Variability

First, in order to illustrate the nature of statistical failure, we have performed creep and constant loading rate (CLR) tests in compression by using the same fluting material randomly chosen. Figure 3 shows the examples of creep curves at a given load, and forcedisplacement curves of CLR tests at a given loading rate. The difference in the stochastic nature of the failure is apparent between these loading histories: the variation of time-to-failure in creep (lifetime) is much greater than the variation of force at failure in CLR tests.

The frequency distribution of creep lifetime obtained at the same applied load is shown in Fig. 4a. It is characterised by a large number of premature failures (short lifetimes) and, at the same time, a

(a)

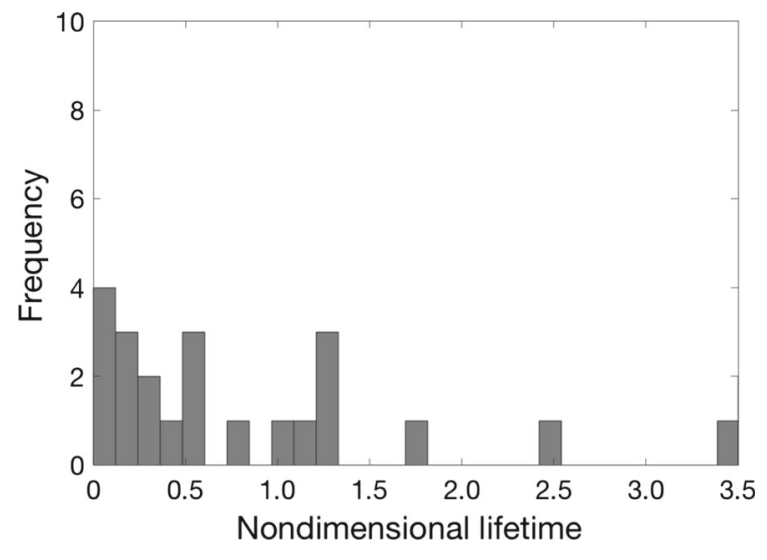

Fig. 4 a Typical frequency distribution of creep lifetime for fluting samples of basis weight $140 \mathrm{~g} / \mathrm{m}^{2}$ (applied load: $56.0 \mathrm{~N}$ ). The lifetime data are made dimensionless by dividing with the persistent tail in the very long lifetime range. Figure $4 \mathrm{~b}$ shows the cumulative distribution function of lifetime, $F\left(t_{B}\right)$ plotted in a Weibull format. The creep lifetime data approximately follow Weibull distribution, as was observed earlier (Mattsson and Uesaka 2013) and also predicted by the current model.

Another important test for the model validation concerns the damage evolution law (Eq. 6). If the damage evolution law is valid, then the model predicts that (1) increasing creep loads horizontally shifts the cumulative distributions in the Weibull format to the left, and (2) when plotted in a log-log format, mean (or median) lifetime vs. applied load relations should be linear. Figure 5a examines the first condition. Individual curves indeed shifted horizontally to the left with increasing applied load. To examine the second condition, the median lifetime values are plotted against applied load in a log-log format in Fig. 5b. The relation was linear, as predicted by Eq. 3, and we observed such linearity consistently for all other sample sets. (The reason for taking median, instead of mean, is that it is difficult to precisely determine the mean lifetime in creep experiments. This is because of the presence of extremely long-live specimens in creep test. Particularly, at low levels of applied load, not all samples fail within the set experimental time period).

Figure 6a shows the frequency distribution of strength obtained by CLR tests at the same loading rate. The data is typically centered around the mean

(b)

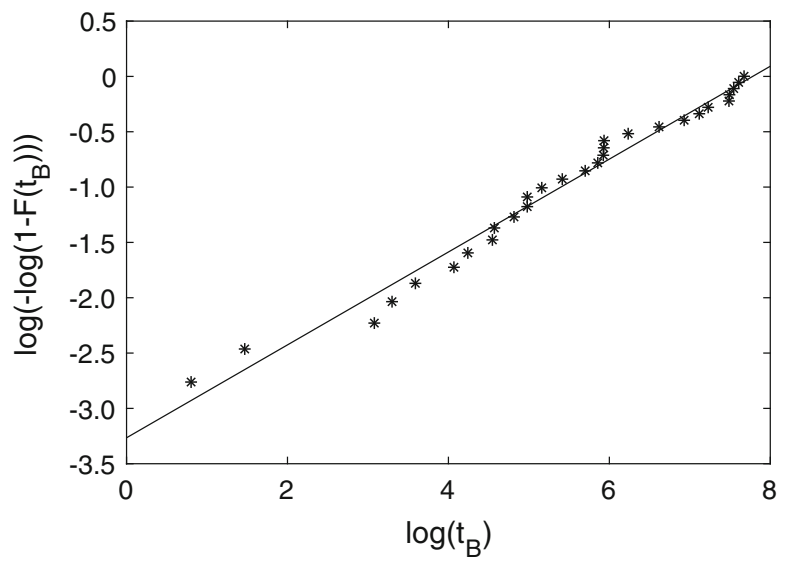

median lifetime. b Cumulative distribution function of the same lifetime data as in (a) plotted in a Weibull format 
(a)

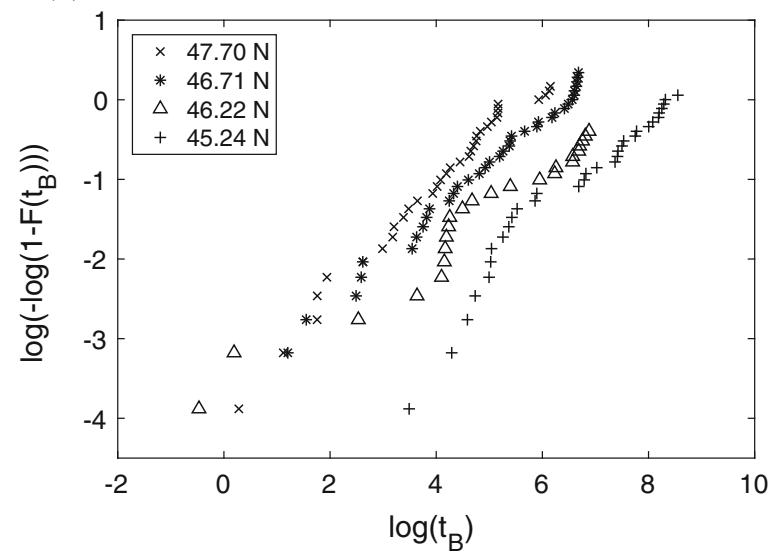

(b)

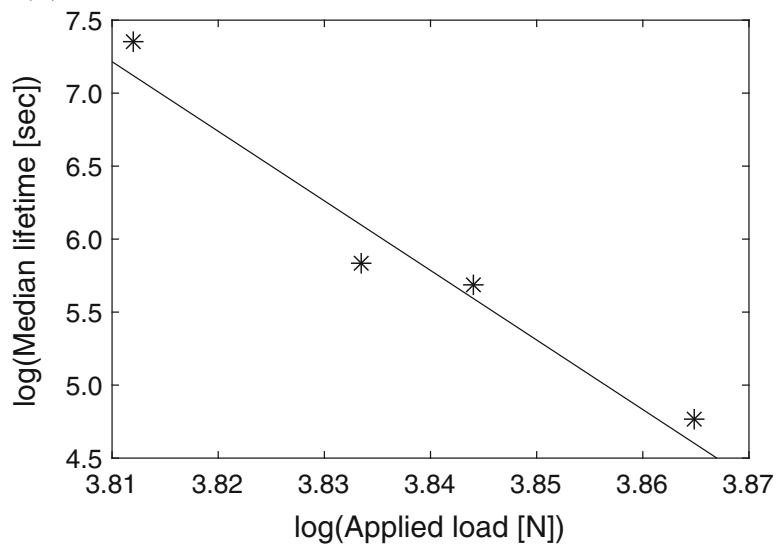

Fig. 5 a Cumulative distribution function of creep lifetime plotted in a Weibull format for four different applied loads (45.24-47.70 N) for linerboard samples of basis weight $135 \mathrm{~g} / \mathrm{m}^{2}$. b Median lifetime versus applied load for the data presented in (a)

(a)

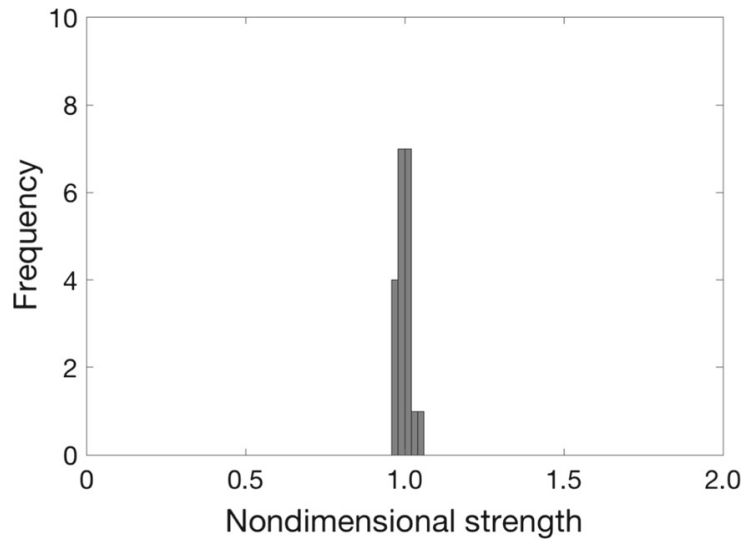

Fig. 6 a Typical frequency distribution of strength measured by constant loading-rate (CLR) tests for fluting samples of basis weight $140 \mathrm{~g} / \mathrm{m}^{2}$ (loading rate: $1 \mathrm{~N} / \mathrm{s}$ ). The strength data are

with a very small scatter, unlike the lifetime distributions. The corresponding data are plotted in a Weibull format in Fig. 6b, which shows, again, Weibull distribution (a straight line in the Weibull plot).

Although both lifetime distributions and strength distributions belong to the same Weibull distribution family, the difference between these distributions reside in their shape parameter (or Weibull exponent), $m$. Coleman (1958) and lately Christensen and coworkers (2009) derived the relationship between the Weibull exponents for lifetime, $m_{\text {creep }}$, and for strength, $m_{\text {strength }}$ : (b)

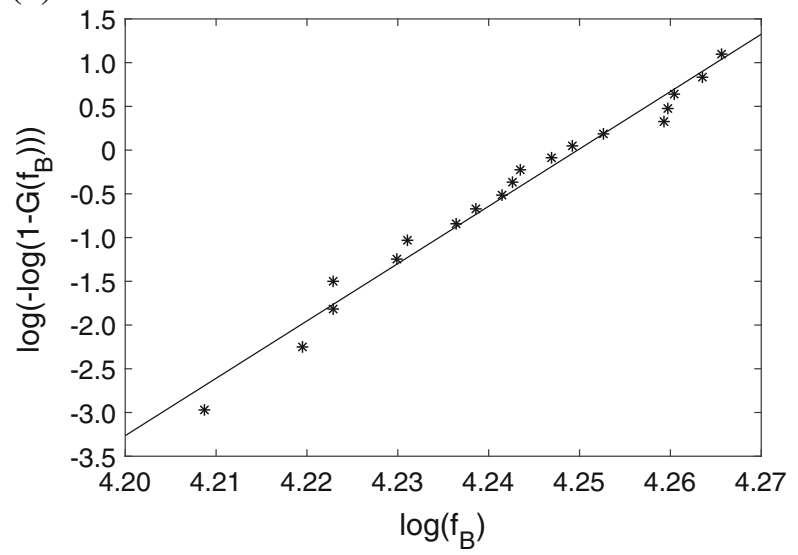

made dimensionless by dividing with the mean strength. b Cumulative distribution function of the same strength data as in (a) plotted in a Weibull format

$m_{\text {creep }}=\beta$ and $m_{\text {strength }}=\beta(\rho+i)$

where $i=1$ or 0 depending on the models. Since $\rho$ is in the order of 20 to 60 in the case of cellulosic materials, it is understandable that the Weibull exponent $m_{\text {strength }}$ is always much higher than $m_{\text {creep }}$. In other words, strength distributions always have much smaller scatters than creep lifetime distributions.

In the case of CLR tests, the model predicts that the strength distributions plotted in a Weibull format are horizontally shifted to the right with increasing loading rates. Figure $7 \mathrm{a}$ indeed showed such trend. It also predicts that taking the mean strength values from 
(a)

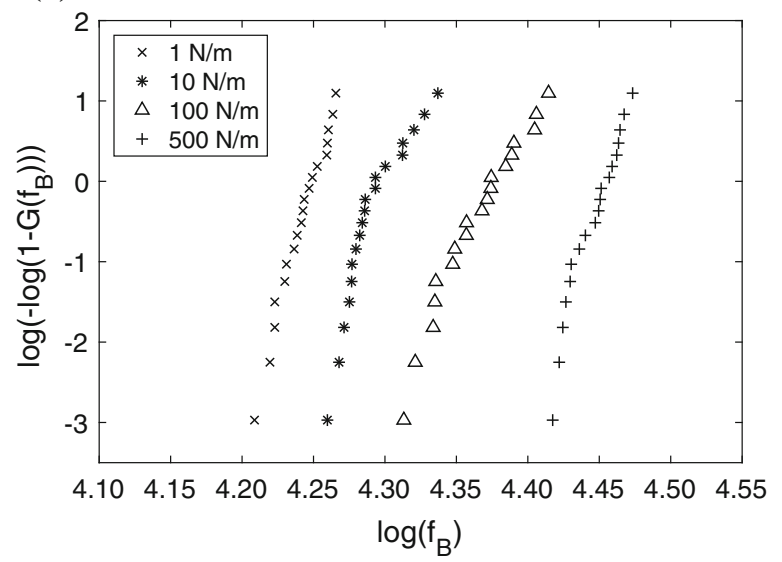

(b)

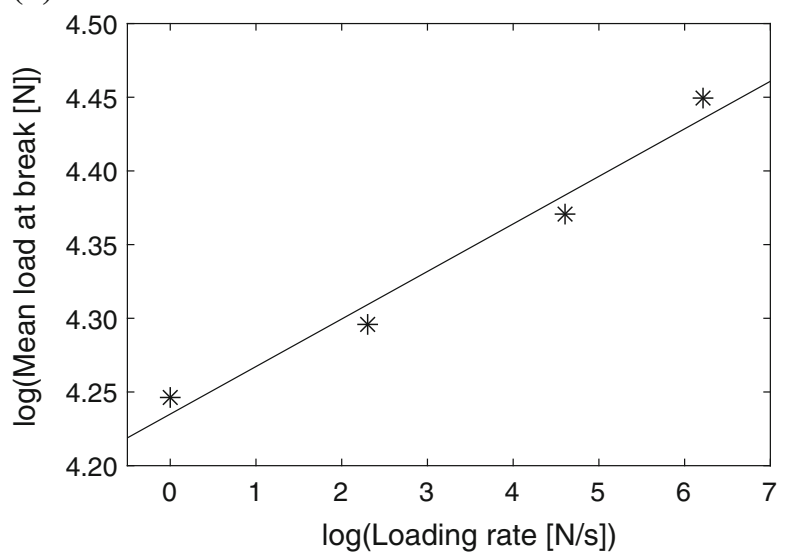

Fig. 7 a Cumulative distribution function of strength plotted in a Weibull format for four different loading rates (1-500 N/s) for fluting samples of basis weight $140 \mathrm{~g} / \mathrm{m}^{2}$. b Mean strength versus loading rate for the data presented in (a)

Fig. $7 \mathrm{a}$ and plotting against the corresponding loading rates yield a linear relationship in the log-log plot. The result (Fig. 7b) precisely showed such relationship.

In summary, the model originally proposed by Coleman describes very well statistical failure responses of cellulosic materials which are subjected to entirely different loading histories, creep and CLR tests.

Characterisation of the material parameters, $S_{c}, \rho$, and $\beta$

We have determined characteristic strength, $S_{c}$, brittleness parameter (or durability parameter), $\rho$, and Weibull shape parameter (or long-term reliability parameter), $\beta$, by performing both creep tests and CLR tests, independently, for a series of commercial containerboard samples (Fig. 8a-c). The error bars in the figures represent the standard error. Although there are large variations among the samples measured by both creep and CLR tests, there is no systematic difference in the results between the creep and CLR tests. Unfortunately, direct comparisons between the creep and CLR tests using the (nominally) same samples were not possible, because of the batch-tobatch and position-to-position variations of the samples, as mentioned earlier in the subsection "Numerical determination of the parameters".

Nevertheless, results showed some interesting characteristics of cellulosic materials. The brittleness parameter, $\rho$, varied from about 20 to 60 , in spite of the fact that the samples consist of essentially the same (a)

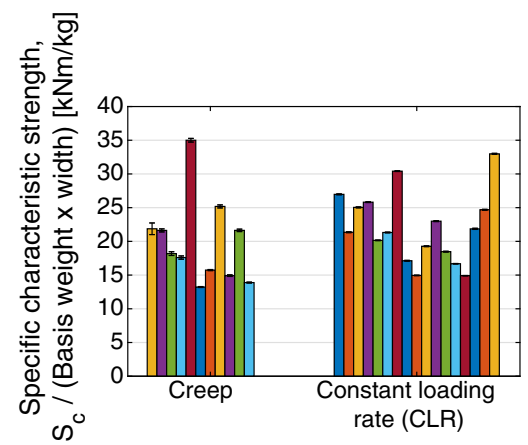

(b)

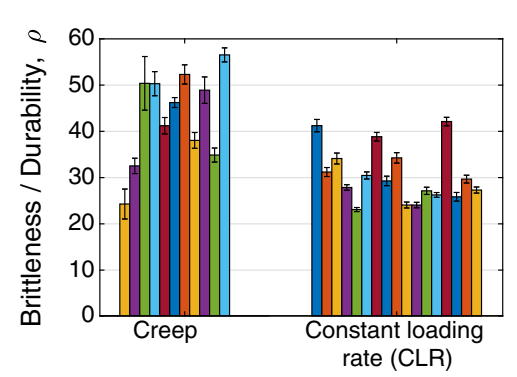

(c)

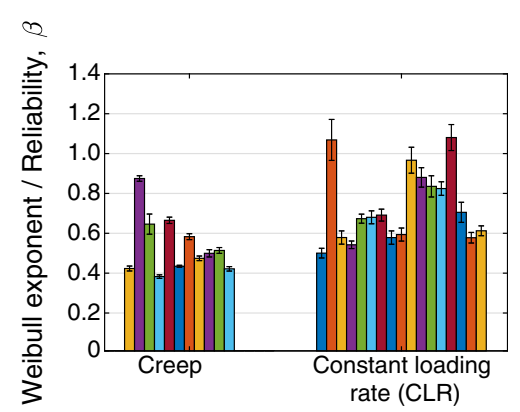

Fig. 8 Material parameters determined by creep and CLR compression tests. a Specific characteristic strength, $S_{c} /$ (basisweight $\times$ width), $\mathbf{b}$ Brittleness parameter (or durability), $\rho$, and $\mathbf{c}$ Weibull shape parameter (or long-term reliability), $\beta$ 
polymer compositions (cellulose, hemicellulose and some residual lignin). In our previous studies (Mattsson and Uesaka 2015, 2017) based on Monte Carlo simulations of time-dependent, statistical failures, the parameter $\rho$ is determined by the element-level (or molecular level) properties, including temperature effects (Phoenix and Tierney 1983), see Eq. 7, rather than the network structures. It would be interesting to see how $\rho$ varies with chemical compositions or moisture content.

Another parameter, reliability, $\beta$, also varied considerably from 0.4 to over 1.0 . This parameter is affected by both fibre and network disorders (Mattsson and Uesaka 2015, 2017). The parameter $\beta$ of the network is directly proportional to the one for the constituent fibres (Mattsson and Uesaka 2017). Therefore, any non-uniformity of fibre will affect the $\beta$ parameter of network. Our previous study also discussed the types of disorders that affect $\beta$ the most. Among the factors, both geometrical disorders (random network structures) and also fibre stiffness variations had significant impacts on the $\beta$ parameter of the fibre network.

Although, in this study, we were not able to compare, directly, the material parameter values obtained from these creep and CLR tests, a few data are available in the literature for carbon-based fibres, glass fibres, Kevlar fibres, and their composites. Results showed excellent agreements of $\rho$ and $\beta$ values measured by the creep tests and the strength tests (constant displacement-rate (CDR) tests (Christensen 2009)).

Lastly, it might be worth to note the CDR tests. Equation 4 requires the constant loading-rate history in order to determine the three material parameters. However, since many of the brittle and quasi-brittle materials show nearly a linear stress-strain response up to failure, one may be tempted to use the CDR tests, instead of the CLR tests. We have compared these two test modes for a limited number of samples for cellulosic materials (containerboards). CDR tests tended to exhibit a rather gradual (or ductile) failure processes, particularly at slow loading (or displacement) rates, say, $1 \mathrm{~N} / \mathrm{s}$. This made the peak-value determination very difficult, as compared with the CLR tests, and thus we were unable to use the CDR tests for the estimation of the material parameters. By considering this uncertainty of the CDR tests, we have determined the upper and lower bounds of the peak values, and compared with the mean strength values and distributions from the CLR tests. The results showed that the CLR results are indeed well bounded by the CDR results. However, in order to justify the use of CDR tests, one still needs a more systematic comparison with the CLR tests.

Comparisons of cellulosic fibre networks with advanced composite materials

We have compared the material parameters, $\beta$ and $\rho$, obtained from our containerboard samples, with other types of fibre materials. Such data are not widely available, but a limited number of data have been reported in the literature for fibre-based materials used for advanced composites (Otani et al. 1991; Phoenix et al. 1988; Phoenix 1978; Farquhar et al. 1989; Wagner et al. 1986), see Fig. 9. There is a clear trend that the higher the $\rho$ value (brittleness), the lower the $\beta$ (reliability). In other words, highly brittle, though durable, materials tend to exhibit large variabilities in long-term performance (e.g., creep lifetime). This relationship was actually predicted in our previous Monte-Carlo simulation studies (Mattsson and Uesaka 2015, 2017). Interestingly, the cellulosic fibre networks used for containerboards showed rather high $\beta$ values (high reliability) with modest $\rho$ values. The values are comparable with other types of fibre materials, such as Kevlar-epoxy and glass-epoxy composites. With enhancing the performance further, these cellulose-based fibre networks may become an even more competitive and favorable material for structural composite applications.

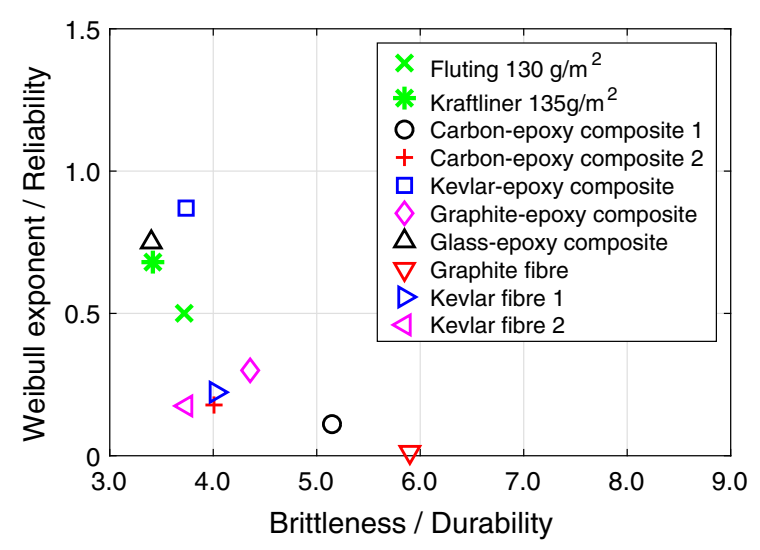

Fig. 9 Comparison of $\beta$ and $\rho$ between containerboard samples and typical fibre-based materials used for structural composites 


\section{Conclusions}

Strength properties of cellulosic materials have been investigated for many decades. However, the aspects of time-dependent, statistical failures, which are commonly seen in fatigue, creep failure, or, more generally, end-use failures of structural members, are still poorly understood. The approaches to handle such properties have been also largely empirical. We have used a general framework of time-dependent, statistical failures, which was originally proposed by Coleman (1958). This theory is phenomenological, but it has been examined and tested intensively by both theoretical analyses and Monte-Carlo simulations. We have demonstrated, experimentally, that this model can be applied also to cellulosic materials, by using creep tests and constant loading rate (CLR) tests. These tests can determine, independently, the three materials parameters, i.e., (1) characteristic strength, $S_{c},(2)$ brittleness parameter (or durability parameter), $\rho$, and (3) Weibull shape parameter (or long-term reliability parameter), $\beta$. Results showed that these two test methods provide comparable values for the material parameters, in spite of the large difference in the measurement time-scale and the differences in equipment geometries. This suggests that, by replacing the traditional creep tests with the CLR tests, one can drastically shorten the testing time for determining the material parameters, say, from months to a few days. Lastly we have also compared the cellulose fibre materials (containerboards) with fibres and composites used for advanced composite structures. We have shown that the cellulosic materials are very competitive in terms of reliability and possess durability comparable with Kelvar and glass fibre composites.

Acknowledgments The financial support provided by the KK-Foundation in Sweden is greatly appreciated. The authors wish to thank Staffan Nyström at Mid Sweden University for the help and assistance for setting up the experiments. Rickard Boman at SCA R\&D is also greatly acknowledged for all help with experimental matters. We acknowledge the valuable discussions, experimental supports, and enthusiasms received from SCA R\&D in Sundsvall, and BillerudKorsnäs at Gruvön mill.

Open Access This article is distributed under the terms of the Creative Commons Attribution 4.0 International License (http:// creativecommons.org/licenses/by/4.0/), which permits unrestricted use, distribution, and reproduction in any medium, provided you give appropriate credit to the original author(s) and the source, provide a link to the Creative Commons license, and indicate if changes were made.

Funding The funding was provided by Stiftelsen för Kunskaps- och Kompetensutveckling.

\section{References}

Bronkhorst C (1997) Towards a more mechanistic understanding of corrugated container creep deformation behaviour. J Pulp Pap Sci 23(4):J174-J181

Christensen R, Miyano Y (2006) Stress intensity controlled kinetic crack growth and stress history dependent life prediction with statistical variability. Int $\mathrm{J}$ Fract 137(1-4):77-87

Christensen R, Miyano Y (2007) Deterministic and probabilistic lifetimes from kinetic crack growth-generalized forms. Int J Fract 143(1):35-39

Christensen RM (2009) A physically based cumulative damage formalism. In: Major accomplishments in composite materials and sandwich structures. Springer, pp 51-65

Coffin D (2011) Engineering Mechanics of Paper and Board Products, chap. Creep and Relaxation. De Gruyter, Berlin

Coleman B (1958) Statistics and time dependence of mechanical breakdown in fibres. J Appl Phys 29(6):968-983

Curtin WA, Scher H (1997) Time-dependent damage evolution and failure in materials. mi. mtheory. Phys Rev B 55:12038-12050. https://doi.org/10.1103/PhysRevB.55. 12038

Duxbury P, Leath P, Beale PD (1987) Breakdown properties of quenched random systems: the random-fuse network. Phys Rev B 36(1):367

Farquhar DS, Mutrelle FM, Phoenix SL, Smith RL (1989) Lifetime statistics for single graphite fibres in creep rupture. J Mater Sci 24(6):2151-2164. https://doi.org/10. 1007/BF02385436

Fellers C, Donner BC (2002) Handbook of physical testing of paper, vol. 1, chap. Edgewise compression strength of paper. Marcel Dekker, New York, pp 481-525

Hashin Z, Rotem A (1978) A cumulative damage theory of fatigue failure. Mater Sci Eng 34(2):147-160

Kellicutt K, Landt E (1951) Safe stacking life of corrugated boxes. Fibre Contain 36(9):28-38

Kirkpatrick J, Ganzenmuller G (1997) Engineering corrugated packages to survive cyclic humidity environments-a case study. In: 3rd International symposium: moisture and creep effects on paper, board and containers, Rotorua, New Zealand, Papro New Zealand (1997)

Koning J Jr, Stern R (1977) Long-term creep in corrugated fiberboard containers. Tappi, Peachtree Corners

Mahesh S, Phoenix S (2004) Lifetime distributions for unidirectional fibrous composites under creep-rupture loading. Int J Fract 127(4):303-360. https://doi.org/10.1023/B: FRAC.0000037675.72446.7c

MATLAB: fitnlm (2017) https://se.mathworks.com/help/stats/ fitnlm.html?requestedDomain=true 
Mattsson A, Uesaka T (2013) Time-dependent, statistical failure of paperboard in compression. In: I'Anson S (ed) Advances in pulp and paper research, transactions of the 15th fundamental research symposium, vol 1 . The pulp and paper fundamental research society, Cambridge, UK, pp 711-734

Mattsson A, Uesaka T (2015) Time-dependent statistical failure of fiber networks. Phys Rev E 92(4):042-158

Mattsson A, Uesaka T (2017) Time-dependent breakdown of fiber networks: uncertainty of lifetime. Phys Rev E 95:053,005. https://doi.org/10.1103/PhysRevE.95.053005

Miner MA (1945) Cumulative damage in fatigue. J Appl Mech 12(3):A159-A164

Monkman FC, Grant NJ (1956) An empirical relationship between rupture life and minimum creep rate in creeprupture tests. Proc ASTM 56:593-620

Moody R, Skidmore K (1966) How dead load, downward creep influence corrugated box design. Package Eng 11(8):75-81

Murakami Y, Endo M (1994) Effects of defects, inclusions and inhomogeneities on fatigue strength. Int $\mathrm{J}$ Fatigue 16(3):163-182

Newman W, Phoenix SL (2001) Time-dependent fibre bundles with local load sharing. Phys Rev E 63:021,507-1021,507-20

Nyman U (2004) Continuum mechanics modelling of corrugated board. Ph.D. thesis, Lund University

Otani H, Phoenix SL, Petrina P (1991) Matrix effects on lifetime statistics for carbon fibre-epoxy microcomposites in creep rupture. J Mater Sci 26(7):1955-1970. https://doi.org/10. 1007/BF00543630

Phoenix S, Schwartz P, Robinson H (1988) Statistics for the strength and lifetime in creep-rupture of model carbon/ epoxy composites. Compos Sci Technol 32(2), 81-120
(1988). https://doi.org/10.1016/0266-3538(88)90001-2. http:// www.sciencedirect.com/science/article/pii/0266353888900012

Phoenix SL (1978) Stochastic strength and fatigue of fiber bundles. Int J Fract 14(3):327-344. https://doi.org/10. 1007/BF00034692

Phoenix SL, Schwartz P, Robinson H (1988) Statistics for the strength and lifetime in creep-rupture of model carbon/ epoxy composites. Compos Sci Technol 32(2):81-120

Phoenix SL, Tierney L (1983) A statistical model for the time dependent failure of unidirectional composite materials under local elastic load-sharing among fibers. Eng Fract Mech 18(1):193-215

Popil RE, Hojjatie B (2010) Effects of component properties and orientation on corrugated container endurance. Packag Technol Sci 23(4):189-202

Stott R et al (2017) Compression and stacking strength of corrugated fibreboard containers. Appita J 70(1):75

TAPPI (2013) T402-Standard conditioning and testing atmospheres for paper, board, pulp handsheets, and related products. Standard, TAPPI

Tierney L (1982) Asymptotic bounds on the time to fatigue failure of bundles of fibers under local load sharing. Adv Appl Probab 14:95-121

Wagner HD, Schwartz P, Phoenix SL (1986) Lifetime statistics for single kevlar 49 filaments in creep-rupture. J Mater Sci 21(6):1868-1878. https://doi.org/10.1007/BF00547921

Wilshire B (2002) Observations, theories, and predictions of high-temperature creep behavior. Metall Mater Trans A 33(2):241-248

Wu B, Leath P (1999) Failure probabilities and tough-brittle crossover of heterogeneous materials with continuous disorder. Phys Rev B 59(6):4002-4010 